\title{
STOP-IT: Windows executable software for the stop-signal paradigm
}

\author{
Frederick VERBRUGGEN \\ Vanderbilt University, Nashville, Tennessee \\ and Ghent University, Ghent, Belgium \\ GORDON D. LOGAN \\ Vanderbilt University, Nashville, Tennessee \\ AND \\ Michä̈l A. STEVENS \\ Ghent University, Ghent, Belgium
}

\begin{abstract}
The stop-signal paradigm is a useful tool for the investigation of response inhibition. In this paradigm, subjects are instructed to respond as fast as possible to a stimulus unless a stop signal is presented after a variable delay. However, programming the stop-signal task is typically considered to be difficult. To overcome this issue, we present software called STOP-IT, for running the stop-signal task, as well as an additional analyzing program called ANALYZE-IT. The main advantage of both programs is that they are a precompiled executable, and for basic use there is no need for additional programming. STOP-IT and ANALYZE-IT are completely based on free software, are distributed under the GNU General Public License, and are available at the personal Web sites of the first two authors or at expsy.ugent.be/tscope/stop.html.
\end{abstract}

The stop-signal paradigm is a useful tool for the investigation of response inhibition. However, programming a stop-signal experiment in popular software packages requires advanced programming skills. Moreover, the latency of the stop process cannot directly be observed and needs to be estimated. To make the stop-signal paradigm more accessible to all researchers, we introduce a free-touse stop-signal task program and an analyzing program that can be installed easily on Windows computers without additional programming.

\section{The Stop-Signal Paradigm}

The stop-signal paradigm (Lappin \& Eriksen, 1966; Logan \& Cowan, 1984; Vince, 1948) was developed to investigate response inhibition in a controlled laboratory setting. In this paradigm, subjects perform a choice reaction task (i.e., the primary task), and, on a random selection of the trials, an auditory stop signal instructs subjects to withhold their response. When the delay between the primarytask stimulus and the stop signal (stop-signal delay, or SSD) increases, the probability of responding on stopsignal trials, $p$ (respond|signal), increases. To account for this observation, Logan and colleagues (Logan \& Cowan, 1984; Logan, Cowan, \& Davis, 1984; see also Boucher, Palmeri, Logan, \& Schall, 2007) proposed a horse-race model, assuming that response inhibition succeeds or fails, depending on the relative finishing time of two processes that race against each other: a go process triggered by the target and a stop process triggered by the stop signal. If the stop process finishes before the go process does, subjects inhibit their response (i.e., signal-inhibit trials). If the go process finishes before the stop process does, subjects fail to inhibit their response (i.e., signal-respond trials).

In addition to accounting for overt performance in the stop-signal paradigm, the horse-race model allows an estimate of the covert latency of the stop process - the stopsignal reaction time (SSRT). This measure has proven useful in cognitive science, cognitive neuroscience, and studies investigating life span development, psychopathology, and individual differences (for reviews, see Boucher et al., 2007; Logan, 1994). For example, several studies have demonstrated that SSRT is elevated in younger children (Williams, Ponesse, Schachar, Logan, \& Tannock, 1999), older adults (Kramer, Humphrey, Larish, Logan, \& Strayer, 1994), impulsive people (Logan, Schachar, \& Tannock, 1997), and children with attention-deficit/hyperactivity disorder (ADHD; e.g., Jennings, van der Molen, Pelham, Brock, Debski, \& Hoza, 1997; Schachar \& Logan, 1990), in comparison with appropriate control groups.

\section{Practical Drawbacks}

Despite its assets, the stop-signal paradigm has some practical drawbacks. First, it is difficult to program, in comparison with other paradigms. Second, unlike with a

F. Verbruggen, frederick.verbruggen@ugent.be 
typical choice reaction task, one of the dependent variables cannot be observed directly and therefore needs to be estimated from calculations based on the horse-race model.

Two features of the stop-signal paradigm complicate the task of programming it: There are multiple overlapping events on stop-signal trials, and multiple SSDs are needed. The stop-signal paradigm requires more than one SSD to discourage subjects from prolonging primary-task RT, thereby decreasing $p$ (respond|signal). To obtain reliable SSRT estimates, researchers have used a staircase tracking procedure: When inhibition is successful, SSD increases by $50 \mathrm{msec}$, but when inhibition is unsuccessful, SSD decreases by $50 \mathrm{msec}$ (see, e.g., Logan et al., 1997; Osman, Kornblum, $\&$ Meyer, 1986). This method results in a $p$ (respond|signal) of .5 and provides the most reliable SSRT estimations (Band, van der Molen, \& Logan, 2003; Logan et al., 1997).

Presenting stop and go stimuli within a trial and implementing a tracking procedure require a flexible software package. Popular software packages, such as E-Prime (www.pstnet.com/products/e-prime), offer this flexibility by allowing inline coding, which requires advanced programming skills. However, not all researchers are sufficiently trained for this task and are thereby forced to use other (and sometimes less suitable) paradigms. To overcome this problem, we developed a program called STOPIT to run the stop-signal paradigm. The main advantage of STOP-IT is that it can be used out of the box, without the need for additional programming. Yet flexibility is offered by means of a configuration file, in which users can change some of the defaults.

We also developed a program called ANALYZE-IT, which provides descriptive statistics, such as mean go RT, accuracy, and $p$ (respond|signal), and provides estimates of SSRT. As outlined above, the horse-race model allows estimation of the covert latency of the stop process. The idea is depicted in Figure 1. The model assumes that $p$ (respond|signal) for a certain SSD corresponds to the area on the left of the vertical dashed line. Given that the primary-task RT distribution and $p$ (respond|signal) are known to the experimenter, it is possible to estimate when the internal response to the stop signal occurs (RTir; see Figure 1). Because the starting point of the stop process is also known (based on the SSD), the experimenter can estimate the SSRT by subtracting SSD from RTir (see Logan, 1994; Logan \& Cowan, 1984, for a more detailed discussion of the horse-race model and of different methods to estimate SSRT).

STOP-IT uses the tracking procedure to adjust SSD throughout the experiment. Under this procedure, subjects will stop half of the responses [i.e., $p$ (respond|signal $)=.5$ ], so RTir must equal the mean go RT (Logan et al., 1997). Consequently, SSRT can be estimated by subtracting mean SSD from mean RT (Logan et al., 1997). This provides SSRT estimates that are derived from the central part of the primary-task RT distribution, and Logan et al. (1997; see also Band et al., 2003) demonstrated that these "central" estimates are most reliable. In addition to estimating SSRT, ANALYZE-IT calculates mean $p$ (respond|signal), mean SSD, mean signal-respond RT, no-signal RT, percentage of correct responses on no-signal trials, and the percentage of missed responses on no-signal trials.

\section{STOP-IT: A STOP-SIGNAL PROGRAM}

STOP-IT can be installed on computers running Windows 2000/XP and is available on the Internet under a GNU license. The software is based on the Tscope library (Stevens, Lammertyn, Verbruggen, \& Vandierendonck, 2006), which was developed to offer accurate timing in Windows 2000/ XP. Tscope functions are used for randomization, primarytask stimulus presentation, stop-signal presentation, ${ }^{1}$ and response registration. In addition, standard $\mathrm{C}$ functions are used for the staircase tracking procedure and for some additional features of the program, such as writing the data to an output file and presenting feedback after each block.

\section{Installation}

The installer is executable in Windows and can be downloaded at expsy.ugent.be/tscope/stop.html or at the

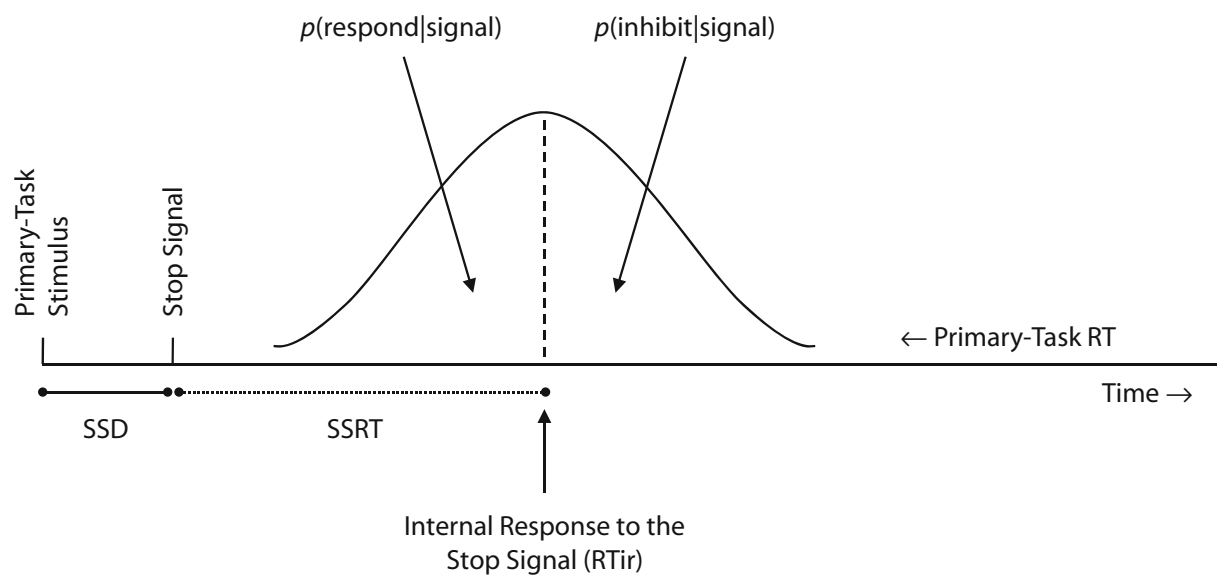

Figure 1. Illustration of the probabilities of responding [ $p$ (respond|signal)] based on the horserace model (Logan \& Cowan, 1984), given the distribution of no-signal reaction times (primarytask RT), the stop-signal delay (SSD), and the stop-signal reaction time (SSRT). $p$ (respond|signal) is represented by the area under the curve to the left of the dashed line. 
personal Web sites of the first and second authors. The program will be installed in the default program folder. In this folder, researchers can also find the output folder (see below) and the original source files. The main advantage of STOP-IT is that it is a precompiled executable file, and, for basic use, there is no need for additional programming. However, as explained in the Instruction file, advanced users can always adjust the source files as desired.

During the installation, different shortcuts are created in the Start menu: shortcuts to STOP-IT and ANALYZEIT, a shortcut for uninstalling the software, a shortcut to the output folder (see below), a shortcut to the configuration file (see below), and, finally, a shortcut to the instruction file. The instruction file will be opened in the default browser and explains how STOP-IT and ANALYZE-IT can be used.

\section{The Stop-Signal Task}

The primary task is a shape judgment task that requires subjects to discriminate between a square and a circle. On no-signal trials ( $75 \%$ of the trials), only the primary-task stimulus is presented, and subjects are instructed to respond to the stimulus as fast and accurately as possible. On stop-signal trials ( $25 \%$ of the trials), the primary-task stimulus is followed by an auditory stop signal, and subjects are instructed to withhold their responses.

Stimuli. The primary-task stimuli are a square and a circle. The fixation sign $(+)$ and stimuli are presented in the center of the screen, in white, on a black background. Stimulus size will depend on the screen size and on graphic parameters that are defined in the configuration file. Occasionally, a stop signal $(750 \mathrm{~Hz}, 75 \mathrm{msec})$ is presented shortly after the stimulus onset in the primary task. Note that the volume of the stop signal will depend on Windows' sounds settings. These settings can be adjusted by clicking on the speaker icon in the notification area or by going to Control Panel $\rightarrow$ Sound and Audio Devices.

Procedure. The program starts when the user enters a subject number. Then the task instructions are presented on the screen. The experiment consists of two phases: a practice phase of 32 trials and an experimental phase of three blocks of 64 trials. As we will outline below, some of these default numbers can be changed.

In both phases, each trial starts with the presentation of the fixation sign, which is replaced by the primary-task stimulus after $250 \mathrm{msec}$. By default, the response keys are " $\mathrm{Z}$ " (for square) and "/" (for circle). The experiment is aborted when the ESC key is pressed. The stimulus remains on the screen until subjects respond, or until 1,250 msec (i.e., the maximal RT) have elapsed. The default interstimulus interval is 2,000 msec and is independent of RT. On stop-signal trials, a stop signal is presented after a variable SSD. SSD is initially set at $250 \mathrm{msec}$ and is adjusted continuously with the staircase tracking procedure: When inhibition is successful, SSD increases by $50 \mathrm{msec}$; when inhibition is unsuccessful, SSD decreases by $50 \mathrm{msec}$. Response registration continues during stop-signal presentation.

Between blocks, subjects have to wait for $10 \mathrm{sec}$ before they can start the next block. During this interval, subjects will receive information about their performance in the last block: the number of incorrect responses on no-signal trials, the number of missed responses on no-signal trials, the mean RT on no-signal trials, and the percentage of correctly suppressed numbers.

Output files. After each block, the results are written to a data file that is stored in the output folder that was created during installation. When the experiment is aborted, the data are written to the data file before the application closes. The data file is a tab-delimited file, stop-X.txt, where $X$ corresponds to the subject number. This file can be opened in Microsoft Excel or in statistical software packages, such as SPSS. The data folder can be accessed easily by using the shortcut in the Start menu (Start $\rightarrow$ All Programs $\rightarrow$ STOP-IT $\rightarrow$ Output Dir). STOP-IT will never overwrite data files; when a data file already exists (i.e., the subject number has already been used), the experimenter is instructed to enter a new subject number. When data files need to be replaced (e.g., because a subject stopped significantly more or less than $50 \%$ of the time; see below), the user can manually delete the output file with the basic file system utilities.

The data file consists of the following information: block number, trial number, stimulus number $(1=$ square; $2=$ circle $)$, trial type $(0=$ no-signal trial $; 1=$ stop-signal trial), whether the response was correct $(0=$ incorrect no-signal trial or signal-respond trial; $2=$ correct nosignal trial or signal-inhibit trial), RT, measuring error (in microseconds), and, finally, SSD. There is one line for every trial.

\section{The Configuration File}

The program can be used immediately after installation. However, users can adjust some of the default values of the program. As explained in the instruction file (Start $\rightarrow$ All Programs $\rightarrow$ STOP-IT $\rightarrow$ Instruction File), this can be done by opening the configuration file in the default text editor (on most computers, this will be Notepad) or in Microsoft Word. To open the configuration file, subjects can click on the shortcut in the Start menu (Start $\rightarrow$ All Programs $\rightarrow$ STOP-IT $\rightarrow$ Configuration File). We refer to the Web site of Tscope (expsy.ugent.be/tscope) for detailed information about the configuration of screen and timing settings.

Users can change four categories of task parameters: the number of practice trials, the number of experimental blocks, the maximal RT, and the initial SSD. Second, users can change various screen parameters, such as screen size, refresh rate, and screen mode (i.e., whether the program is run in a window or fullscreen). Third, users can change timing parameters, such as the priority of the program (high priority increases timing precision). Finally, users can change the response keys. It should be noted that the first key defined is always mapped onto square, the second key defined is always mapped onto circle, and the third key defined is always the abort key.

Users can change these parameters by replacing the current parameter with one of the other possible parameters that are defined in the configuration file. After the user has saved the file, STOP-IT will automatically use the new parameters; recompiling or reinstalling is unnecessary. 
The configuration file does not allow users to change the primary task or the primary-task stimuli. Users who are interested in manipulating tasks or task variables that are not described in the configuration file will need to make changes in the original source code (Start $\rightarrow$ All Programs $\rightarrow$ STOP-IT $\rightarrow$ stop_task.c). As explained in the instruction file, developmental tools can be downloaded for free, and these tools allow users to make the required changes in the source files and recompile the program. Note that after recompiling the program, the shortcuts in the Start folder can still be used, as long as the file names of the source files are not changed.

\section{ANALYZE-IT: AN ANALYZING PROGRAM}

During the installation of STOP-IT, ANALYZE-IT is also installed. ANALYZE-IT allows users to estimate SSRT for each subject. In addition, it will calculate the means for all dependent variables of interest. Of course, users can still analyze the raw data files themselves.

\section{Usage}

ANALYZE-IT is installed (and uninstalled) together with STOP-IT. During the installation, a shortcut will be created in the Start menu (Start $\rightarrow$ All Programs $\rightarrow$ STOPIT $\rightarrow$ ANALYZE-IT). By clicking on this shortcut, the analyzing software is started.

When ANALYZE-IT is started, the number of subjects $(N)$ must be entered. The program will automatically start with analyzing stop-1.txt and look for all $N$ output files. When an output file is missing (e.g., $N=5$, but stop-3.txt is missing), the program will skip this file and automatically process the next file. For the number of blocks in the experiment, ANALYZE-IT uses the number of blocks defined in the configuration file used by STOP-IT. Note that the first trial of every block is never analyzed.

Dependent variables. The following variables are estimated or calculated: mean $p$ (respond|signal), mean SSD, SSRT, mean signal-respond RT, no-signal RT, percentage of correct responses on no-signal trials, and the percentage of missed responses on no-signal trials.

When the tracking procedure is used, SSRT can be calculated by subtracting mean SSD from the untrimmed mean RT (Logan et al., 1997). Therefore, ANALYZE-IT calculates the mean raw RT first (i.e., the mean RT for all no-signal trials), and then mean SSD is subtracted from this value. ANALYZE-IT will also calculate a $z$ score and the corresponding $p$ value to indicate whether each subject inhibited significantly more or less than $50 \%$ of the time. These values can be found in the output file as well. The subtraction method is not for subjects who inhibited significantly more or less than $50 \%$ of the time, so they must be replaced, or SSRT must be calculated by another method (see Logan, 1994; Logan \& Cowan, 1984). When users replace a subject, they should rename the original data file or delete it (see above).

For each subject, the dependent variables will be written to a tab-delimited file, results.txt, which can be opened in Microsoft Excel or SPSS. Results.txt is stored in the default output folder, which can be accessed by using the shortcut in the Start menu (Start $\rightarrow$ All Programs $\rightarrow$ STOPIT $\rightarrow$ Output Dir). Attentive users will notice that SSRT does not correspond to mean no-signal RT (as reported in the output file) minus the mean SSD. The reason for this discrepancy is that mean signal-respond RT and mean no-signal RT are calculated after the removal of incorrect responses (i.e., when the wrong key is pressed).

\section{CONCLUSIONS}

The stop-signal paradigm is a useful tool for the investigation of response inhibition in a controlled laboratory setting. The stop-signal paradigm is difficult to program, so the present work was designed to make the paradigm more accessible to researchers. We provide programs that can be used to run the stop-signal task (STOP-IT) and to analyze the raw data and estimate the covert latency of the stop process (ANALYZE-IT). STOP-IT and ANALYZEIT are both distributed under the GNU General Public License (Free Software Foundation, 1991) and are available at expsy.ugent.be/tscope/stop.html or at the personal Web sites of the first two authors.

\section{AUTHOR NOTE}

F.V. is a Postdoctoral Fellow of the Research Foundation-Flanders (FWO-Vlaanderen) and is currently at the Department of Psychology at Vanderbilt University. This research was also supported by Grant BCS 0446806 from the National Science Foundation and Grant FA9550-07-1-0192 from the Air Force Office of Scientific Research to G.D.L. We thank Rich Carlson and two anonymous reviewers for comments on a previous version of this article. Correspondence concerning this article should be sent to F. Verbruggen (users.ugent.be/ fvrbrugg/) or G. D. Logan (www.psy.vanderbilt.edu/faculty/logan/), Department of Psychology, Vanderbilt University, Nashville, TN 37203 (e-mail: frederick.verbruggen@ugent.be or gordon.logan@vanderbilt.edu).

\section{REFERENCES}

Band, G. P. H., van der Molen, M. W., \& Logan, G. D. (2003). Horserace model simulations of the stop-signal procedure. Acta Psychologica, 112, 105-142.

Boucher, L., Palmeri, T. J., Logan, G. D., \& Schall, J. D. (2007). Inhibitory control in mind and brain: An interactive race model of countermanding saccades. Psychological Review, 114, 376-397.

Jennings, J. R., van der Molen, M. W., Pelham, W., Brock DebSKI, K., \& HozA, B. (1997). Inhibition in boys with attention deficit hyperactivity disorder as indexed by heart rate change. Developmental Psychology, 33, 308-318.

Kramer, A. F., Humphrey, D. G., Larish, J. F., Logan, G. D., \& Strayer, D. L. (1994). Aging and inhibition: Beyond a unitary view of inhibitory processing in attention. Psychology \& Aging, 9, 491-512.

LAPPIN, J. S., \& ERIKSEN, C. W. (1966). Use of a delayed signal to stop a visual reaction-time response. Journal of Experimental Psychology, 72, 805-811.

LoGAN, G. D. (1994). On the ability to inhibit thought and action: A user's guide to the stop signal paradigm. In D. Dagenbach \& T. H. Carr, Inhibitory processes in attention, memory, and language (pp. 189-239). San Diego: Academic Press.

Logan, G. D., \& Cowan, W. B. (1984). On the ability to inhibit thought and action: A theory of an act of control. Psychological Review, 91, 295-327.

Logan, G. D., Cowan, W. B., \& Davis, K. A. (1984). On the ability to inhibit simple and choice reaction time responses: A model and a method. Journal of Experimental Psychology: Human Perception \& Performance, 10, 276-291.

LOGAN, G. D., SchaCHAR, R. J., \& TANNOCK, R. (1997). Impulsivity and inhibitory control. Psychological Science, 8, 60-64. 
Osman, A., Kornblum, S., \& Meyer, D. E. (1986). The point of no return in choice reaction time: Controlled and ballistic stages of response preparation. Journal of Experimental Psychology: Human Perception \& Performance, 12, 243-258.

ScHACHAR, R., \& LogAn, G. [D.] (1990). Are hyperactive children deficient in attentional capacity? Journal of Abnormal Child Psychology, 18, 493-513.

Stevens, M., Lammertyn, J., Verbruggen, F., \& VandierenDONCK, A. (2006). Tscope: A C library for programming cognitive experiments on the MS Windows platform. Behavior Research Methods, 38, 280-286.

Verbruggen, F., \& De Houwer, J. (2007). Do emotional stimuli interfere with response inhibition? Evidence from the stop signal paradigm. Cognition \& Emotion, 21, 391-403.

Verbruggen, F., Liefooghe, B., Notebaert, W., \& VandierenDONCK, A. (2005). Effects of stimulus-stimulus compatibility and stimulus-response compatibility on response inhibition. Acta Psychologica, 120, 307-326.

Verbruggen, F., Liefooghe, B., \& Vandierendonck, A. (2006a). The effect of interference in the early processing stages on response inhibition in the stop signal task. Quarterly Journal of Experimental Psychology, 59, 190-203.

Verbruggen, F., Liefooghe, B., \& VAndierendonck, A. (2006b). Se- lective stopping in task switching: The role of response selection and response execution. Experimental Psychology, 53, 48-57.

Verbruggen, F., Logan, G. D., Liefooghe, B., \& VAndierendonck, A. (2008). Short-term aftereffects of response inhibition: Repetition priming or between-trial control adjustments? Journal of Experimental Psychology: Human Perception \& Performance, 34, 413-426.

VincE, M. A. (1948). The intermittency of control movements and the psychological refractory period. British Journal of Psychology, 38, 149-157.

Williams, B. R., Ponesse, J. S., Schachar, R. J., Logan, G. D., \& TANNOCK, R. (1999). Development of inhibitory control across the life span. Developmental Psychology, 35, 205-213.

\section{NOTE}

1. The Tscope functions that handle the stop-signal presentations have been used successfully in a number of recent studies performed in our lab (Verbruggen \& De Houwer, 2007; Verbruggen, Liefooghe, Notebaert, \& Vandierendonck, 2005; Verbruggen, Liefooghe, \& Vandierendonck, 2006a, 2006b; Verbruggen, Logan, Liefooghe, \& Vandierendonck, 2008).

(Manuscript received September 24, 2007; revision accepted for pubication December $7,2007$. 